\title{
Essai d'utilisation du microscope électronique à balayage pour l'étude morphologique des champignons
}

\author{
par M. THIBAUT et M. ANSEL \\ Laboratoire de Parasitologie et de Mycologie ( $\mathrm{P}^{r}$ M. LARIVIÈRE) \\ U.E.R. des Cordeliers, 15, rue de l'Ecole-de-Médecine, F $75-$ Paris-6 $^{\circ}$
}

\section{Résumé}

Des culiures du Rhizopus equinus ont été étudiées au microscope électronique à balayage. Cette nouvelle technique a permis d'apporter d'intéressants renseignements.

\section{Summary}

Cultures of Rhizopus equinus were studied with the scanning electron microscope. This new technique has provided interesting information.

Le but de cette note est de signaler l'utilité de l'examen en microscopie électronique à balayage comme élément descriptif pouvant faciliter la détermination, toujours difficile, des diverses espèces de Mucoraceae. Il n'est pas douteux que les caractères biologiques tels que l'utilisation des glucides, les produits du métabolisme (sucres ou alcools) ont une grande importance. Mais dans l'emploi des seuls caractères morphologiques, trop souvent on a recours à des critères tels que la dimension des pedicelles, des sporanges... comme aussi à la présence ou à l'absence de chlamydospores, sans préciser les caractères de celles-ci - Vuillemin semble avoir été le premier à signaler que l'aspect plissé de l'épispore des sporangiospores du Rhizopus caractérisait assez bien ce genre. 


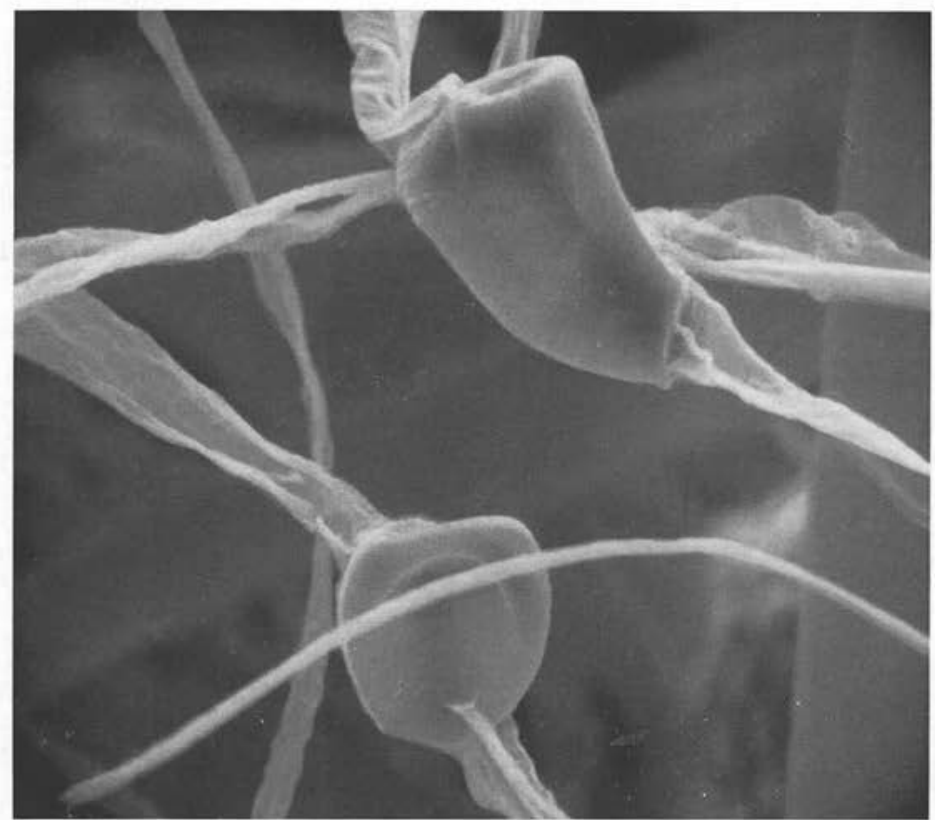

FIG. 1. - Chlamydospores du Rhizopus equinus. Noter leur aspect absolument lisse $(X 2.000)$

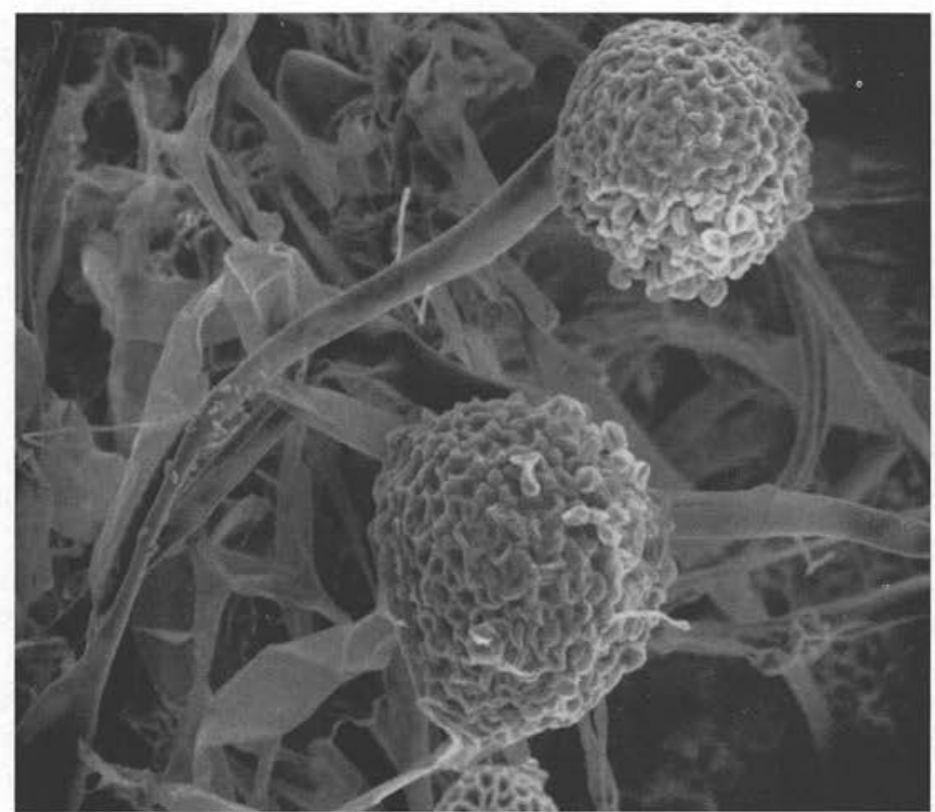

Fig. 2. - Sporanges du Rhizopus equinus. Remarquer leur texture externe $(\times 600)$ 


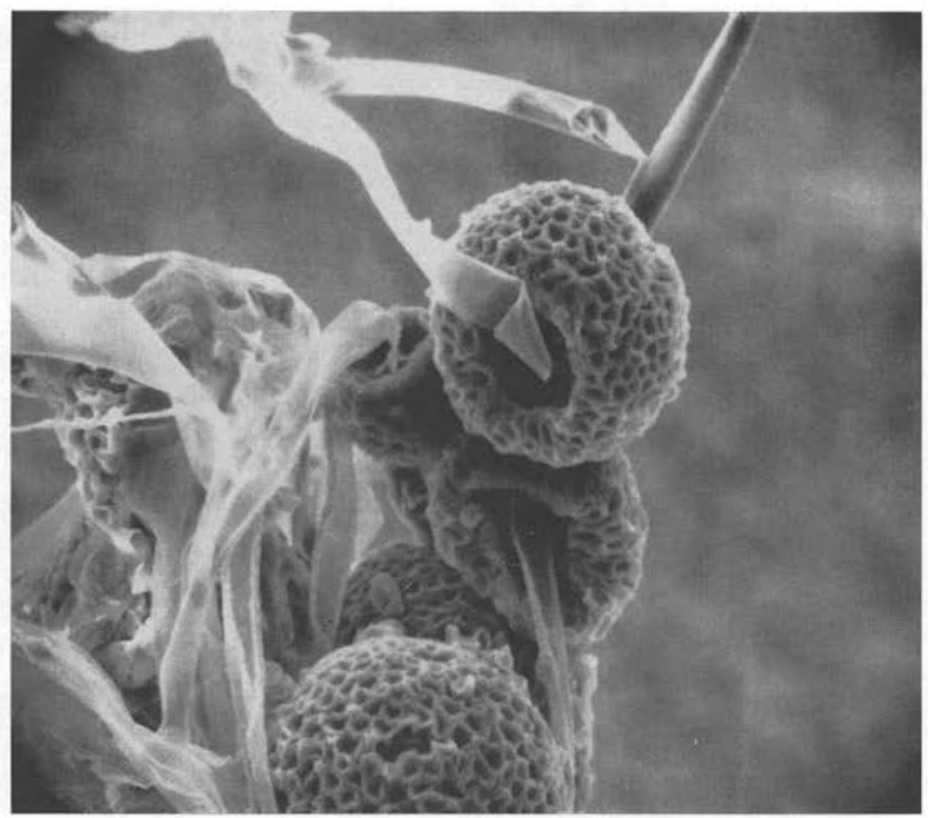

FIG. 3. - Sporanges du Rhizopus equinus. $\times 500$

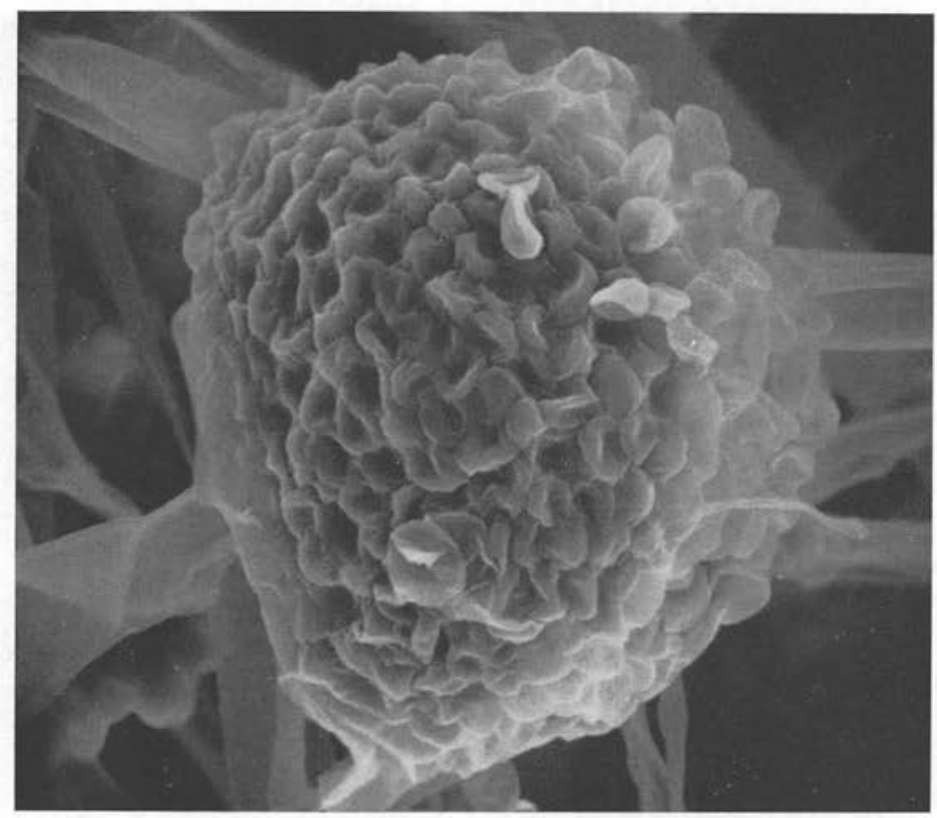

FIg. 4. - Sporange du Rhizopus equinus. $\times 1.200$ 


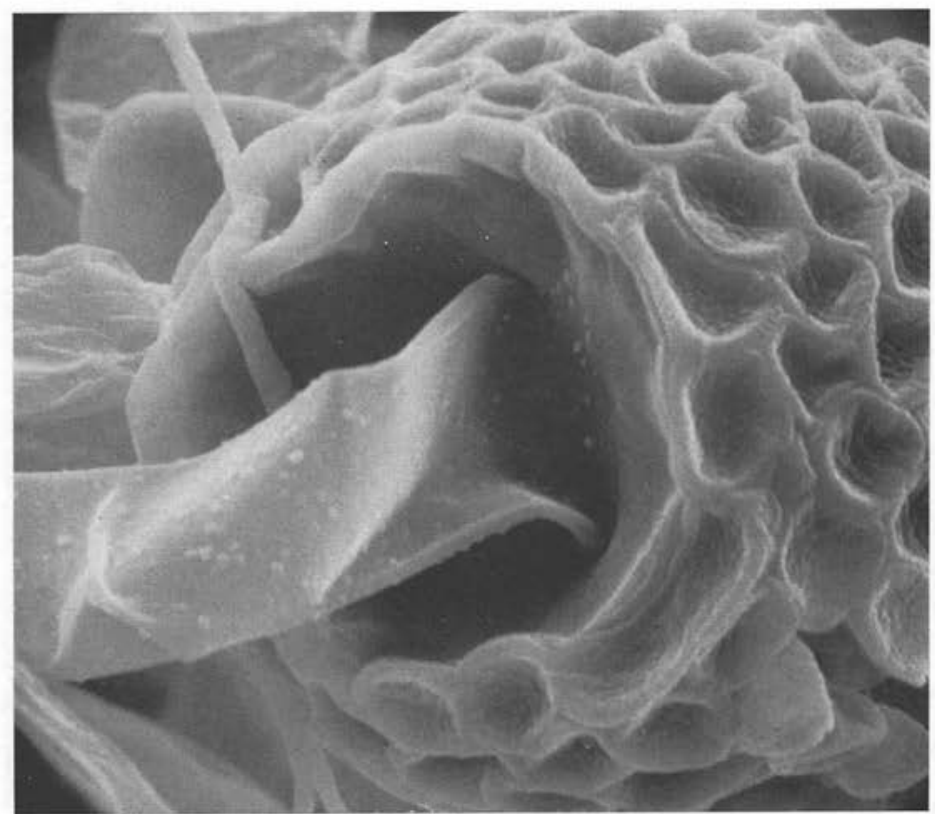

FIG. 5. - Vue inférieure d'un sporange du Rhizopus equinus. Noter les alvéoles de la paroi externe $(\times 3.000)$

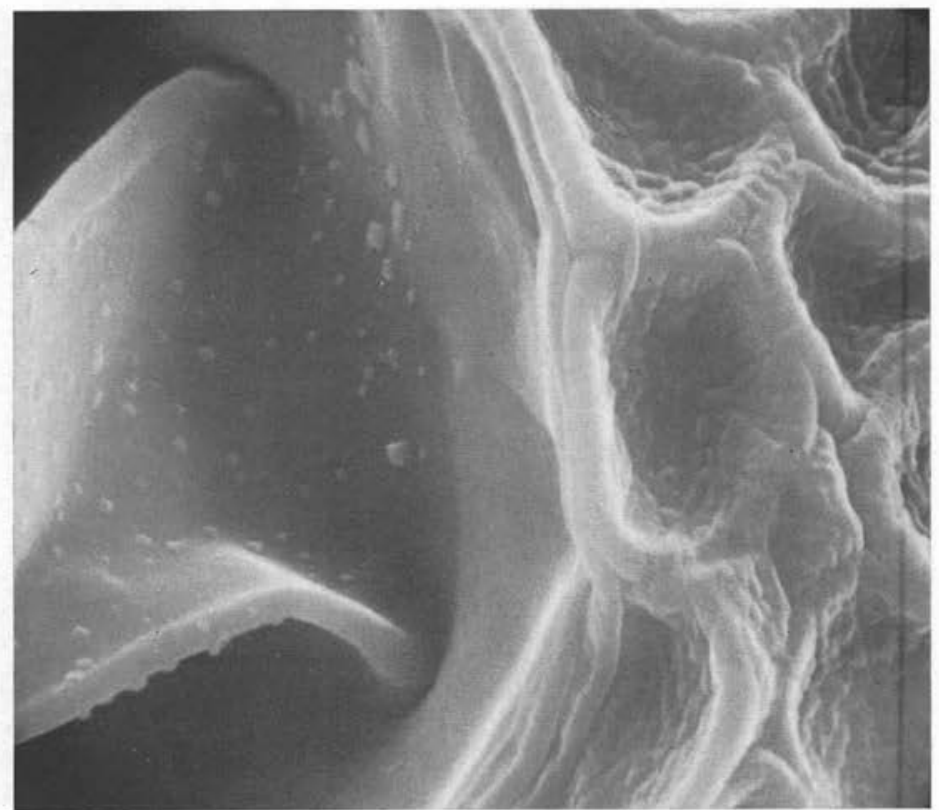

FIG. 6. - Sporange du Rhizopus equinus. Détail de la photo précédente (X 6.200) 
L'examen au microscope électronique à balayage (JSM-U3 JEOLCO) du Rhizopus equinus nous a permis de préciser d'une part l'aspect absolument lisse des chlamydospores (photo 1), d'autre part la remarquable texture externe des parois des sporanges (photo 2), A ce sujet, nous avons noté dans la littérature que les dessins des sporanges du Rhizopus equinus montrent comme vues par transparence à l'intérieur du sporange, les spores qui y sont incluses. La mensuration des alvéoles nous permet de constater que celles-ci ont environ $6 \mu$ de diamètre du sommet d'une crête à un autre, alors que les spores n'ont que 3 à $4 \mu$. Il est vraisemblable que, sans le savoir, les auteurs ont dessiné l'ornementation externe des sporanges, en croyant dessiner les spores.

Quoi qu'il en soit, cette ornementation remarquable nous paraît devoir être signalée et il nous semble que la description de cette structure externe des sporanges devrait être précisée, tout autant que celle des spores, pour la détermination des espèces. Dans cette perspective, l'utilisation de l'examen en microscopie électronique à balayage nous paraît devoir être une technique désormais nécessaire (photos 3, 4, 5, 6).

\section{Bibliographie}

Bernard (P.-N.), 1914. - Sur un Rhizopus pathogène de l'homme. Bull. Soc. mycol. France, 30, 230-232.

Costantin et Lucet, 1903. - Sur un Rhizopus pathogène. Bull. Soc. mycol., France, 29, 200-216.

Van Tieghem (Ph.), 1875. - Nouvelles recherches sur les Mucorinées. Ann. sc. Nat., 6, 5-175.

Vuillemin (P.), 1903. - Importance taxinomique de l'appareil zygosporé des Mucorinées. Bull. Soc. mycol., France, 29, 106-118.

-, 1931. - Les champignons parasites et les mycoses de l'homme, Lechevalier ed., Paris. 